Journal of Accident and Emergency Medicine 1995

12, 115-118

\title{
Treatment of knee sprains: modified Robert Jones or elastic support bandage?
}

\author{
D.L. HUGHES \& A.C. CROSBY \\ Department of Accident and Emergency Medicine, Royal Hallamshire Hospital, Sheffield, UK
}

\section{S U M M A R Y}

The aim of this investigation was to compare in a prospective randomized study the outcome of painful, traumatic knee injuries when treated with either the modified Robert Jones (MRJB) or elastic support bandage (ESB). Patients with moderate or severe unilateral knee injury presenting to our department within $24 \mathrm{~h}$ were randomized into two treatment groups receiving either the MRJB or an ESB. The main outcome parameters of the study were the amount of pain relief required, the speed of recovery, mobility and patient preference. The results of our study of $\mathbf{4 0}$ patients indicate that the two treatments were equally effective in treating knee sprains, and patients preferred the ESB in the early post-injury period. Therefore, we see no reason to continue using the MRJB for the treatment of sprained knees in the accident and emergency (A\&E) setting when a more patient acceptable, time and cost-effective treatment is available.

Key words: elastic support bandage, knee sprain, modified Robert Jones bandage.

\section{INTRODUCTION}

The treatment of traumatic knee sprains in A\&E departments is poorly documented and often governed more by hearsay and tradition than by rational proven treatment regimes. The 'Robert Jones' bandage, first described by Major Robert Jones in 1915, ' and later in more detail by Charnley ${ }^{2}$ consisted of many layers of calico and wool, and was used to apply pressure to joint effusions and to offer controlled limitation of movement. The bandage has been modified over the years and now consists of three layers of crepe interspersed with two layers of wool.

This 'Modified Robert Jones' bandage (MRJB) has become a standard treatment for knee sprains, along with the elastic support bandage (ESB). The decision to use one rather than the other is the subject of this investigation.

We designed a prospective randomized controlled study to compare the two treatments. In this study, we adopted the null hypothesis, assuming that there was no significant difference in our measured parameters between the MRJB and the ESB. The MRJB is more expensive ( $\$ 10$ compared with 50p), time consuming (20 min compared with $5 \mathrm{~min}$ ) to assemble, less comfortable, and makes no difference to the delay in return to normal activity.

\section{SUBJECTS AND METHODS}

This study included all patients presenting during a 3-month period to a teaching hospital A\&E department receiving $\mathbf{5 5 0 0 0}$ new cases per year. All patients with moderate or severe unilateral knee injury presenting within $24 \mathrm{~h}$ were considered. All patients were examined carefully, and those without any signs or symptoms attributable to an acute internal derangement of the knee (history of 'giving way', locking, or signs of cruciate ligament or meniscal tears) were selected and randomized into two treatment groups. Each patient received an advice sheet giving instructions on mobilization, and a walking stick.

The following categories were excluded from the trial:

(1) congenital abnormality;

(2) a fracture in either lower limb;

(3) any patient rendered incapable of weight bearing;

(4) joint instability (i.e. ligamentous tear);

(5) lipohaemarthrosis (swelling appearing immediately);

(6) blood dyscrasia;

(7) current/recent steroid or anticoagulant therapy;

(8) previous problems with that knee, including surgery; 
D.L. Hughes \&

A.C. Crosby
(9) locked knee, or any case requiring urgent orthopaedic referral;

(10) evidence of internal derangement, as described above.

Signed consent was obtained from and an explanatory letter given to each patient. Patients were then randomized into one of the two treatment groups. Those patients with an odd A\&E number were given a MRJB and patients with an even A\&E department number were given an ESB.

\section{Patient treatment}

The MRJB used in our department consisted of a layer of 6-inch crepe bandage, a layer of wool, another layer of crepe, a second layer of wool, and a final layer of crepe. The ESB used was Tubiton, one of the commercially available brands.

All patients were given a walking stick and prescribed analgesics, Co-dydramol, two tablets as required up to four times a day. All patients were informed that they would be reviewed at weekly intervals until the knee had recovered fully. It has been shown that routine 'blanket' referral for physiotherapy is unjustified and wasteful of resources in the treatment of knee sprains. ${ }^{3}$

\section{Patient follow-up}

At each patient visit, a standard form was completed in order to obtain the following information:

(1) visual-analogue score for pain;

(2) range of movement of the knee;

(3) number of analgesics taken in the previous week;

(4) degree of comfort/patient preference (very good [5]/good/fair/poor/very poor[1]).

\section{Statistical analyses}

For each of the selected criteria, at each attendance, a Mann/Whitney $U$-test for unpaired, non-parametric data was performed. P-values were obtained from standard published tables. The results were considered to be significant if $P<0.05$.

\section{RESULTS}

A total of $\mathbf{4 0}$ patients were included in this study to compare the effectiveness of the MRJB and ESB. Patients were randomized as shown in Table 1.
Table 1. Number of patients participating in MRJB vs. ESB study

\begin{tabular}{rrr}
\hline Timer & MRJB & ESB \\
\hline Week 0 & 26 & 14 \\
Week 1 & 20 & 11 \\
Week 2 & 10 & 5 \\
Week 3 & 4 & 1 \\
\hline
\end{tabular}

\section{Pain score}

A visual analogue score was used to compare pain at the time of the injury and for 3 weeks thereafter with the treatments indicated. These results are shown in Fig. 1. The null hypothesis was adopted for each week, assuming there was no difference in pain scores. This was accepted at week 0 $(P=0.4009)$, at week $1(P=0.316)$, and at weeks 2 and $3(P>0.4)$. We conclude therefore that the patients were drawn from the same population, and that there was no significant advantage, in terms of pain, of one treatment over the other.

\section{Range of movement}

Range of movement of the knee was assessed at the time of injury and at the weekly follow-up clinic using a goniometer. These results are shown in Fig. 2. Again, the null hypothesis was adopted, but this time at week 0 it was rejected $(P=0.026)$, demonstrating that those patients randomized to

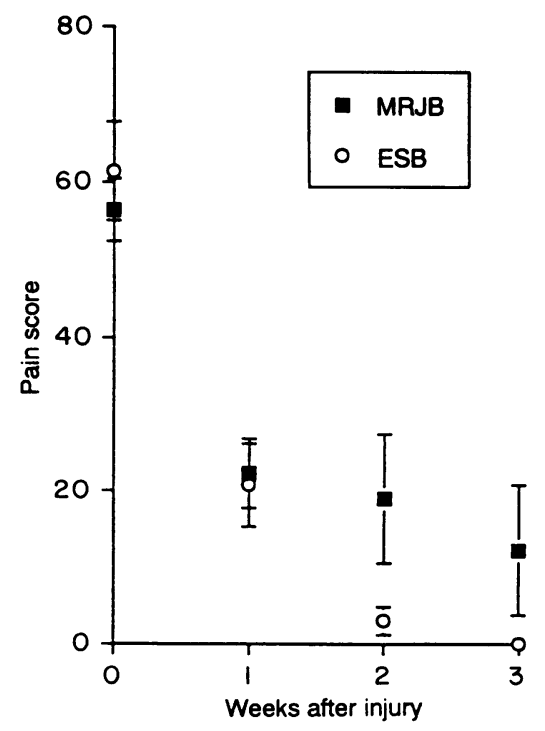

Fig. 1. Pain scores for MRJB and ESB. 
Treatment of knee sprains

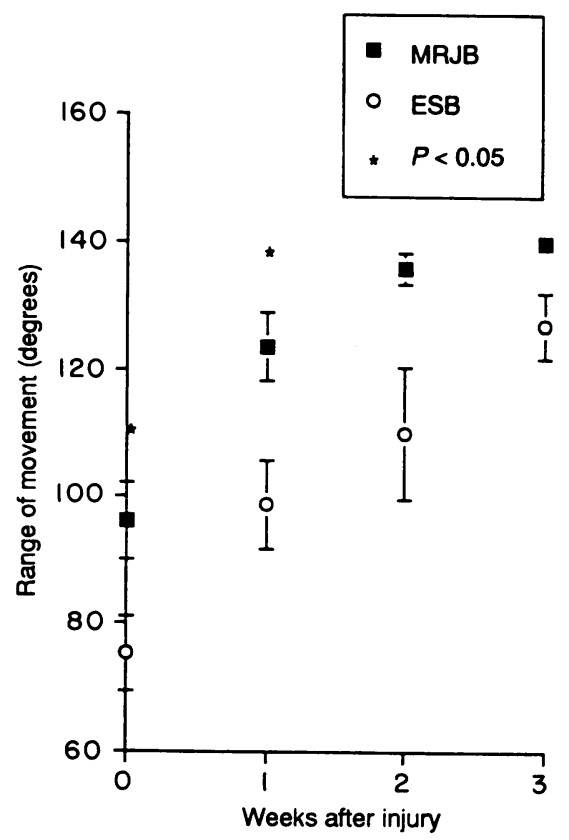

Fig. 2. Range of movement for MRJB and ESB.

the ESB treatment arm showed more limitation of movement. This trend continued at the first weekly review, but thereafter, the null hypothesis was accepted, there being no significant difference in joint mobility (week 2, $P=0.12$; week $3 P>0.4$ ).

\section{Analgesic consumption}

Patients were asked to record their analgesic consumption during the weeks after injury. These results are shown in Fig. 3. The null hypothesis assumes no difference between the two treatment arms, and this is borne out by our analysis (week $1, P=0.47$; week $2, P=0.71$ ).

\section{Patient satisfaction}

Patients were also asked to record whether they found their bandage comfortable, using a scale of 1-5 where $1=$ poor and $5=$ very comfortable. The results are shown in Fig. 4. After the first week, patients preferred the ESB (null hypothesis rejected; $P=0.026$ ). Thereafter, there was no preference (null hypothesis accepted; $P>0.5$ ).

\section{DISCUSSION}

There have been no studies reported in the literature to date which systematically compare the treatment of knee sprains. Our study is the first of its kind to compare the MRJB and ESB.

Two patients recruited to the trial were referred to the orthopaedic surgeons at their second followup appointment and were therefore excluded from the results presented here. Both had been treated with modified Robert Jones bandages. One required intensive physiotherapy, and the other needed repair of a partially torn anterior cruciate ligament.

Both patient groups in this study display a similar increase in range of movement of the knee joint after the initial injury, together with a decreasing pain score over the treatment period of 3 weeks.

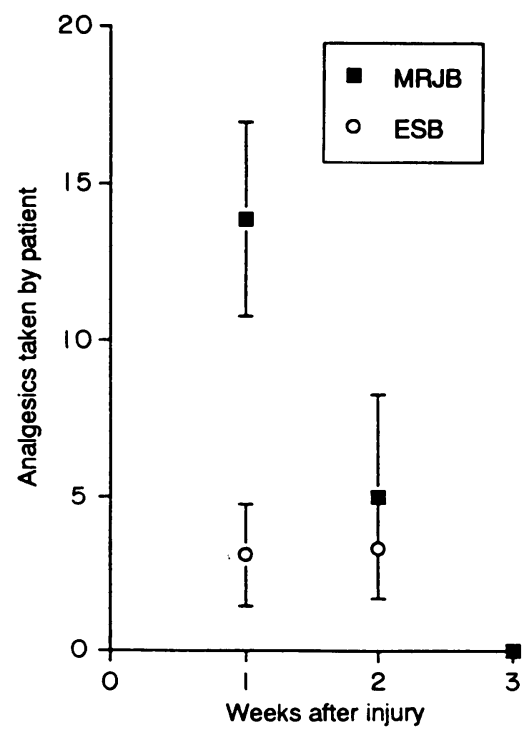

Fig. 3. Analgesics required with MRJB and ESB.

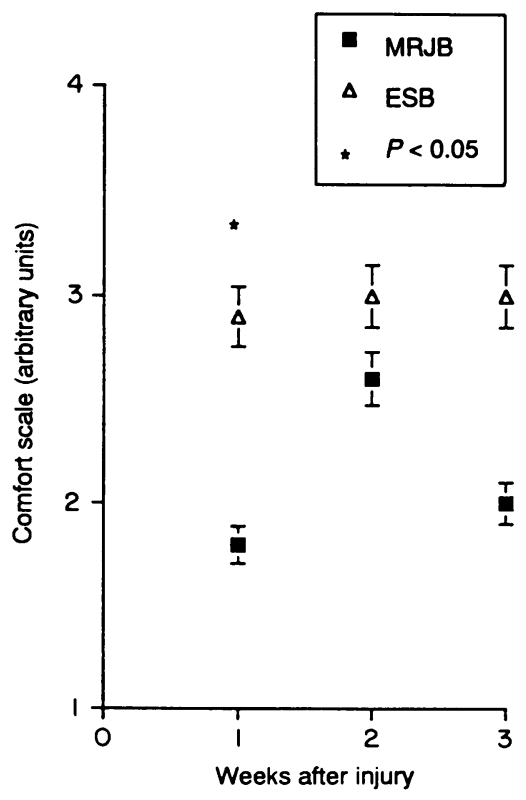

Fig. 4. Patient preference for MRJB or ESB. 
D.L. Hughes \&

A.C. Crosby
The analgesic usage by the two groups is not significantly different at any time point in the study. It is clear from this investigation that patient comfort is significantly increased with the ESB at 1 week although, since the numbers involved are small, this trend is lost in subsequent weeks. Clinicians have long suspected that patients treated with MRJB remove them, and reapply them for their clinic visit, to please us!

Since the numbers involved in our study are small, we hope to accumulate further data to enable more reliable statistical analysis to be performed. For all the criteria deemed important before the start of the study, namely pain control and speed of recovery, the two treatments were equally satisfactory. In terms of patient satisfaction, the ESB was preferred.

In recent years, the MRJB has become popular for treating post-traumatic knee injuries. However, it has been shown by Rosen and Schatzker (1983) at an AO/ASIF conference that, although pressure transducers placed under a Robert Jones bandage (RJB) exhibit a high initial pressure, this persists for only $5 \mathrm{~min}$ or less, rendering the bandage useless. This finding has been supported ${ }^{4}$ and contradicted by other groups, ${ }^{5}$ who claim that the pressure exerted by the RJB stays high for approximately $24 \mathrm{~h}$, declining thereafter, and depends on the technique of application.

For these reasons, and at a time when cost is an increasingly important consideration, we see no reason to continue using the modified Robert Jones bandage in the treatment of sprained knees in the Accident and Emergency setting.

\section{ACKNOWLEDGEMENTS}

The authors would like to thank the medical and nursing staff of the Accident and Emergency Department of the Royal Hallamshire Hospital, Sheffield, for their assistance with this work.

\section{REFERENCES}

1. Jones R. (1921) Injuries of joints. Orthopaedic Surgery of Injuries Oxford University Press, London.

2. Charnley J. (1950) The treatment of fracture without plaster of Paris. In: Closed Treatment of Common Fractures, pp. 28-29. Churchill Livingstone, Edinburgh.

3. Pickard M.A., Venner R.M., Ford I. \& Todd B.D. (1990) The influence of immediate physiotherapy in the outpatient management of acute knee injuries: a controlled study. Archives of Emergency Medicine 7, 249-252.

4. Bentley G. (1984) Current concepts of etiology and treatment of chrondromalacia patellae. Clinical Orthopaedics and Research 189, 209-228.

5. Brodel D.J., Axon D.L. \& McCollister Evarts C. (1986) The Robert Jones bandage. Journal of Bone and Joint Surgery 68, 776-779. 